\title{
Del perfil electoral a la interacción con la ciudadanía. La apropiación de Twitter por los agentes del Parlamento de Galicia
}

Xabier Martínez-Rolán, Universidade de Vigo - xabier.rolan@uvigo.es

Teresa Piñeiro-Otero, Universidade da Coruña - teresa.pineiro@udc.es

Resumen

El salto de la esfera política convencional a la Web ha implicado la aparición de nuevos espacios y fórmulas para la relación entre agentes políticos y ciudadanía. Desde una perspectiva de comunicación y participación política, resulta particularmente destacable Twitter por su carácter público y su trascendencia social. Esta plataforma de microblogging actúa como un canal online que supone casi una prolongación de la esfera pública, y donde representantes y representados establecen nuevos tipos de interacción basados en nuevas reglas. Se trata de un nuevo canal para la participación de la ciudadanía y el contacto de los actores políticos con ésta. El presente artículo ha tenido por objeto analizar la presencia, influencia y participación de los diputados del Parlamento de Galicia en Twitter. Un análisis que ha tenido la finalidad de determinar en qué medida los actores políticos se valen de esta plataforma social como un espacio para "escuchar» las demandas de la ciudadanía gallega, a la que representan. Asimismo, el estudio desarrollado ha permitido señalar diferentes niveles de apropiación de la plataforma tanto por diputados individuales como por grupos parlamentarios.

Palabras clave

Twitter, redes sociales, participación, política, representación, Parlamento de Galicia.

Sumario

1. Introducción; 2. Agentes políticos y participación 2.0.; 3. Metodología; 4. Desigual presencia de diputados y grupos parlamentarios; 5 . Fechas de creación de los perfiles. Para la campaña y más allá; 6 . Actividad de los perfiles y perfiles activos; 7 . Influencia; 8 . A modo de conclusión; 9. Bibliografía. 


\section{Intoducción}

Internet ha dado un vuelco al modo de entender la comunicación política (Gibson y Römmele, 2007). La transposición de la comunicación política convencional a la Web ha conllevado su redimensión especialmente en lo que atinge a tres aspectos: la aparición de nuevas formas no institucionales de política, el establecimiento de una red interconectada de usuarios y la emergencia de la «autocomunicación de masas» (Castells, 2009).

Las actuales formas de cultura política se desligan de los procesos de comunicación de las instituciones, para formar parte de un entorno altamente complejo, de convergencia de viejos y nuevos medios (Rueda Ortiz, 2009).

En este contexto, siguiendo a Cabrera (2009), los social media constituyen una oportunidad única para la construcción de opinión en el entorno digital en tanto que configuran formas de interacción social definidas a través de intercambios de carácter dinámico (Rizo, 2003). Hablar de política 2.0 supone hacerlo de una nueva forma de entender las relaciones entre gobernantes y gobernados (Matud Juristo, 2009) en unas plataformas que constituyen una expansión de la esfera pública (Westling, 2007).

Frente a la opinión pública agregada expresada a través de medios, sondeos y urnas, en el ámbito digital adquiere relevancia la opinión pública discursiva, producto emergente de una conversación colectiva en expansión, enmarcada en el ámbito de la sociedad civil (Resina de la Fuente, 2010).

El ciudadano 2.0 tiene interés por la política, pero sobre todo por aquella movilización que se desarrolla al margen de las instituciones clásicas. Este individuo, que en la Red asume un rol activo y se manifiesta preocupado por el contexto que lo rodea, presenta una frustración y decepción por la política en la arena real (Gutiérrez-Rubí y Antoni, 2010). Como señalan Anduiza et altres (2010a y 2010b) frente al $29 \%$ de internautas españoles que han interactuado en la Red con asociaciones y organizaciones sociales, solamente el $5 \%$ afirma haberlo hecho con algún partido político.

Casacuberta y Gutiérrez-Rubí (2010) subrayan la existencia de dos tendencias divergentes: la propensión a un mayor activismo en la Red y el creciente desinterés por la política oficial como reflejan la elevada abstención electoral y la emergencia de nuevas fórmulas como Escaños en blanco o Podemos. Dos tendencias que constituyen ambas caras de una moneda: la existencia de una brecha cada vez más amplia entre lo emergente y renovador, y su supuesta representación política (Casacuberta y Gutiérrez-Rubí, 2010).

En este contexto, las redes sociales están ejerciendo como plataformas de politización de demandas dado su uso personal para canalizar intereses concretos que generan los niveles más fuertes de interacción (Resina de la Fuente, 2010). Más allá de las plataformas sociales para promover iniciativas de ciberactivismo (como AVAAZ, change.org, Purpose, 38 Degrees, Get Up! o Move On), las redes sociales generalistas se han convertido en espacios políticos en tanto permiten una apertura a las ideas y acciones de nuevos sujetos que generan influencia a través de procesos de contagio (González-Bailón, 2009).

Asimismo desde la perspectiva de los agentes políticos convencionales el acceso a las plataformas sociales no solo facilita la proximidad con la ciudadanía; también permite en cierta medida la igualación de las grandes fuerzas políticas con aquellas minoritarias 0 no consolidadas, dada la ausencia de filtros como los impuestos por los media (Padró-Solanet y Carenal, 2008).

La Red posee múltiples potencialidades para la comunicación política que puede llevar a cotas de expresión y participación inimaginables en un contexto convencional. En este sentido el presente trabajo ha tenido por objeto analizar el grado de apropiación de Twitter, como canal de participación más próximo y directo con la ciudadanía, por los agentes del Parlamento de Galicia.

\section{Agentes políticos y participación 2.0.}

El desarrollo de la Sociedad de la Información en España ha llevado a diversos sujetos y estructuras del ámbito de la política a incorporar las herramientas 2.0 a sus prácticas cotidianas, en diversos grados e intensidades (Araya Dujisin, 2005: 58). Ya en 2010 el Informe sobre política y Redes Sociales ponía de relevancia la alta valoración de las redes sociales por los políticos españoles, 8 de cada 10 las consideraba un método excelente para llegar a la ciudadanía. Asimismo, alrededor de 9 de cada 10 internautas ratificaban dicha valoración al considerar el traspaso de los agentes políticos a las redes sociales como algo necesario en términos de proximidad e interacción. No obstante, mientras miles de sujetos y entidades han logrado apropiarse de las plataformas 2.0 para el activismo social, en el caso de los partidos políticos todavía constituye una asignatura pendiente.

Esta divergencia ha llevado a Borge (2005) a referirse a la existencia de distintas lógicas de los actores colectivos también en el uso de las TIC. Parlamentos, ejecutivos, partidos políticos, etc. pretenden reforzar la democracia representativa pero sin ampliar los canales de participación; mientras que la sociedad civil aboga por otro tipo de democracia más participativa.

La utilización de Internet y las TIC para canalizar demandas ciudadanas ha presionado a las instituciones y actores políticos para modernizar de sus estructuras, crear espacios propios en la Web, la disposición de la información en abierto para el acceso de los usuarios y la apertura de nuevos canales para la participación (Araya Dujisin, 2005).

Cada vez más, los Parlamentos y sus agentes amplían su presencia online a nuevos canales y servicios 2.0 para aproximarse a la sociedad e integrar la participación ciudadana a su actividad. Los estudios sobre e-participación destacan especialmente el papel de las redes sociales en la transparencia de los procesos políticos, la implicación de la ciudadanía o la calidad en la formación de la opinión pública abriendo espacios para la información y la deliberación (Reig, 2012). 


\section{Metodología}

Con la finalidad la presencia de los diputados del Parlamento de Galicia en Twitter y el grado de apropiación de dicha plataforma se ha desarrollado un análisis cuantitativo-cualitativo de sus perfiles en esta red social un año después de la cita electoral autonómica del 21 de octubre de 2012 (en adelante 210).

Se parte de la hipótesis de que en este momento los representantes gallegos habrán normalizado su actividad como diputados, buscando nuevos canales de conexión con la ciudadanía, sin que su actividad se vea afectada por la proximidad de otros comicios como los del Parlamento Europeo (Mayo 2014). La selección de la red de microbloging radica en su carácter público que condicionará tanto el modo de comunicar como el tipo de mensaje vehiculado.

Durante el primer año de la IX Legislatura -en el que se inscribe este análisis- los 75 representantes de la ciudadanía gallega en la Primera Institución gallega se organizaban en cuatro grupos parlamentarios: Grupo Popular (41 escaños), Grupo Socialista (18 escaños), Alternativa Galega de Esquerdas, AGE (Coalición electoral de de ANova Irmandade Nacionalista y de Esquerda Unida-Izquierda Unida con 9 escaños) y el Bloque Nacionalista Galego (BNG, con 7 escaños) ${ }^{1}$. De los 75 diputados autonómicos solamente 36 han formado parte de la muestra de análisis en tanto que disponen un perfil propio en la red de microblogging y, por tanto, de un canal de interacción accesible para la ciudadanía a la que representan.

Una vez determinada la muestra final, y recopilados los datos de actividad de dichos perfiles (recopilación efectuada entre el 1 y el 3 de noviembre) se llevó a cabo un análisis en torno a las siguientes variables: número de seguidores de los perfiles, repercusión (índice klout², porcentaje de tuits que son retuiteados por otros usuarios, porcentaje de tuits que son marcados como favoritos), actividad (publicaciones/día, porcentaje de publicaciones que son retuits, utilización de hashtags o de links), interacción con otros usuarios (menciones y porcentaje de respuestas).

Además de la información recopilada de los propios perfiles también se utilizaron otros datos que, sobre éstos, aportaron herramientas de medición online como Klout (http:// Klout.com), Tweet Reach (http://tweetreach.com), Twitonomy (http://www.twitonomy.com) o Tweet Grader ( http://tweet.grader.com).

\section{Desigual presencia de diputados y grupos parlamentarios}

El análisis de la presencia de los diputados en Internet subraya la representación desigual de las fuerzas políticas de la Cámara. Como refleja el Gráfico 1 el porcentaje de perfiles de los diputados autonómicos en esta Red social no sólo no respeta la distribución del hemiciclo, sino que inclusive la invierte.

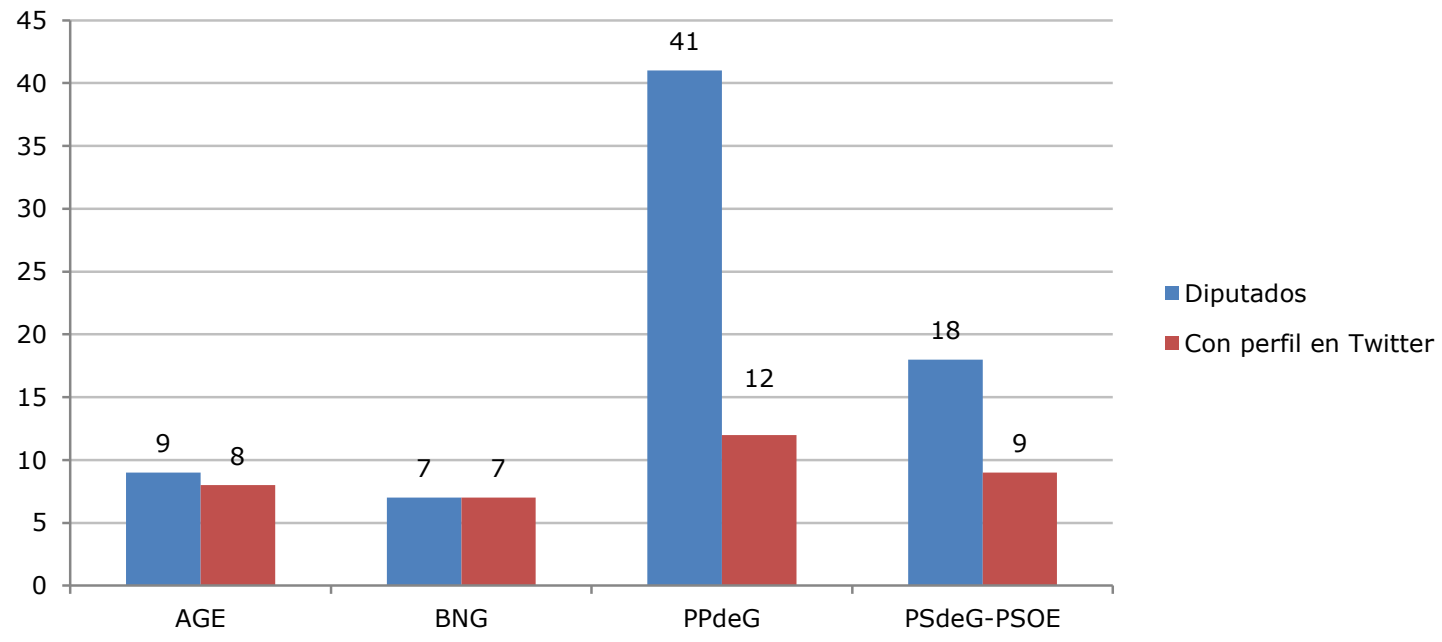

Gráfico 1. Diputados del Parlamento de Galicia en Twitter, por Grupo Parlamentario. Elaboración propia.

Frente al $29 \%$ de los diputados populares o el $50 \%$ de los socialistas con presencia en la plataforma de microblogging, destaca el $92 \%$ de los parlamentarios de AGE que cuentan con su propio perfil en Twitter y el 100\% de los del Bloque Nacionalista Galego (en adelante, BNG).

Esta mayor presencia de los grupos parlamentarios minoritarios en Twitter puede responder a diversas causas como una mayor proyección hacia estas plataformas por límites de acceso a los medios de comunicación clásicos o una estructura de organización más dinámica que facilita la proximidad a la ciudadanía (más compleja en estructuras jerarquizadas).

${ }^{1}$ Los datos del estudio se han recogido en el momento previo a la crisis de Alternativa Galega de Esquerdas, abierta tras la marcha de David Fernández Calviño (diputado de AGE por la provincia de Ourense por parte de ANova), que finalizó con la creación del Grupo Mixto el 10 de febrero de 2014.

2 Índice que se construye a partir de los datos de la cuenta concreta del diputado/a en cuestión, los temas que trata más habitualmente, cómo habla de ellos y cómo reaccionan los usuarios a dicho discurso. 
Otra cuestión reseñable, que ya había sido tratada en un estudio anterior de Piñeiro-Otero y Martínez-Rolán (2013), es el elevado porcentaje de perfiles de Diputados del Parlamento de Galicia que omiten cualquier referencia a su papel en la Cámara. Solamente un $58 \%$ de los perfiles analizados incorporan en la bio de Twitter información relativa a su papel de representantes en la Cámara. Dicha presentación refuerza, en algunos casos, el carácter privado o profesional de la cuenta como un espacio de participación.

\section{Fechas de creación de los perfiles. Para la campaña y más allá}

En lo que respecta a la fecha de creación, en 2009 se abrieron un 9\% de los perfiles de Twitter analizados, un porcentaje similar a 2010 (11\%). En 2011 dicho porcentaje se amplió hasta el 22\% lo que supuso un importante incremento, en la línea de la implantación de dicha plataforma social en el ámbito español. Pero el salto cualitativo se produjo en el 2012, año en el que se crearon más de la mitad de las cuentas de los parlamentarios gallegos en Twitter (47\%).

Este exponencial incremento en 2012 se puede asociar a la inminente cita con las urnas, que llevó a los candidatables a ampliar cuentas en Twitter. De hecho, salvo @RojoNoguera, cuya creación coincide con el inicio de la segunda legislatura, todo los perfiles de diputados que comenzaron su andadura en 2012 lo hicieron con anterioridad al 210 (cita electoral) ${ }^{3}$. Más de la mitad de estos perfiles (9 de 16) fueron creados tras el anuncio anticipado de la convocatoria electoral (27 de agosto de 2012).

Durante el año 2013 se registraron cuatro nuevos diputados en esta plataforma social, lo que supuso un incremento del $11 \%$ de los perfiles de representantes del Parlamento de Galicia en Twitter.

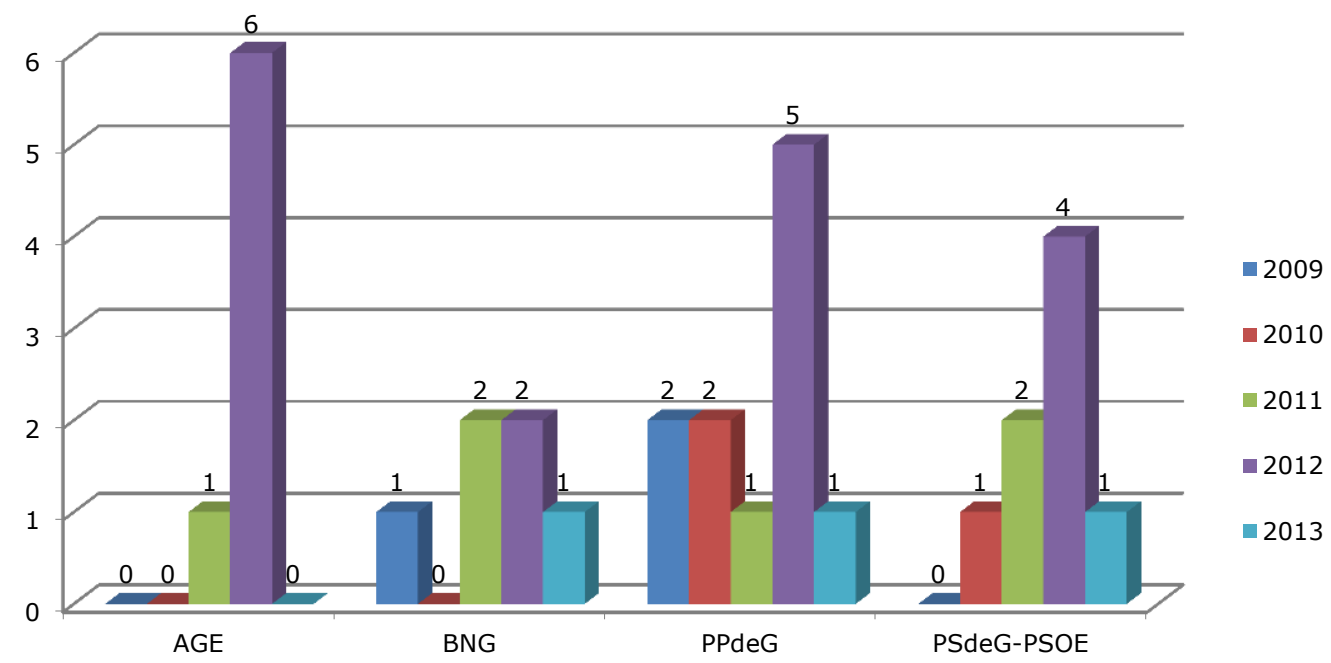

Gráfico 2. Año de creación del perfil en Twitter por grupo parlamentario. Elaboración propia

Atendiendo a la fecha de creación de perfiles por grupos parlamentarios es constatable que, salvo en el caso del BNG, todas las fuerzas de la cámara incrementaron ostensiblemente su presencia en Twitter durante el año 2012 (Gráfico 2).

En el caso de AGE, el exponencial incremento de sus diputados durante 2012 (8 de los 9 diputados crearon su perfil en 2012) debe vincularse al particular contexto de los partidos que forman esta coalición. En concreto, ANova Irmandade Nacionalista fue fundada el 14 de julio de 2012 tras su escisión del Bloque Nacionalista Galego, de modo que el único perfil en Twitter anterior a esta fecha, el de @Chelo_Martínez, fue creado en el momento en que dicha diputada era militante del BNG.

El relevante incremento de las cuentas de parlamentarios AGE durante el año 2012 contrasta con la ausencia de perfiles de nueva creación en 2013. AGE es el único grupo parlamentario que no ha aumentado su presencia en el último año. Un incremento que, en el caso del grupo parlamentario BNG, lo ha consolidado como el único grupo con un $100 \%$ de presencia en Twitter.

\section{Actividad de los perfiles y perfiles activos}

Como señala Gutiérrez-Rubí (2010) en el ámbito 2.0 lo relevante no es tanto estar como fomentar la participación, interacción y la moderación. En este sentido se ha analizado la actividad de los representantes del Parlamento de Galicia en Twitter desde dos vías: los perfiles activos y la actividad de los perfiles.

Son los grupos parlamentarios con menor representación en la cámara quiénes detentan el mayor porcentaje de perfiles activos; todos sus deputados/as han publicado algún tuit en las dos últimas semanas (21 de octubre y el 3 de noviembre de 2013).

\footnotetext{
${ }^{3}$ Resulta preciso destacar la existencia de dos perfiles del diputado socialista Francisco Caamaño: @francaamano y @fran_caamano. La última publicación del primero de ellos data del 13 de noviembre de 2012. Posteriormente el político continuó su actividad en @fran_caamano.
} 


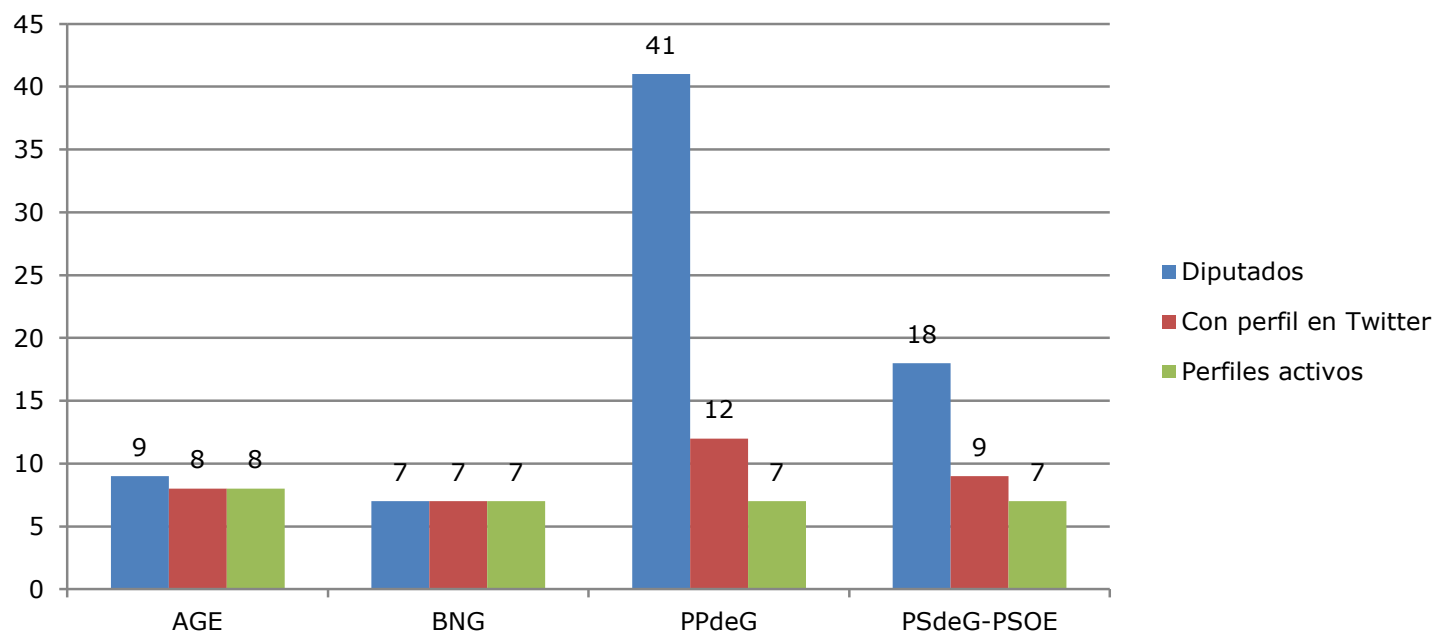

Gráfico 3. Diputados del Parlamento de Galicia con perfil activo en Twitter, por Grupo Parlamentario. Elaboración propia.

Entre los perfiles inactivos resulta destacable la presencia de los candidatos del PSdeG-PSOE y del PPdeG a la Presidencia de la Xunta de Galicia, una cuestión que remite a una utilización del perfil exclusivamente electoral. Una utilización que si bien está marcada en la cuenta del Popular Alberto Núñez Feijoo, @feijoo2012 (Twitter oficial da candidatura de Alberto Núñez Feijóo á Presidencia da Xunta de Galicia. As súas mensaxes persoais van asinadas con ANF), no sucede lo mismo con el Socialista Pachi Vázquez, @pachivazquez, cuya última actualización data de abril de 2013.

También resulta preciso mencionar el perfil en Twitter de la presidenta del Parlamento de Galicia, Pilar Rojo Noguera (@RojoNoguera) creado para este rol Institucional al inicio de la IX Legislatura y que no sólo carece de actividad, sino que ésta es privada.

En lo que respecta a la actividad de los perfiles ésta se ha analizado atendiendo a tres variables: publicaciones día, promedio de menciones e índice de respuesta.

Si se parte de la concepción más básica de actividad de un perfil de Twitter, entendida como el número de publicaciones/día se puede observar que 21 de los parlamentarios gallegos con perfil en la plataforma de microblogging publican más de un tuit al día (Tabla 1).

\begin{tabular}{|l|l|l|}
\hline Perfil & Grupo & $\mathbf{N}^{\mathbf{0}}$ de tweets/día \\
\hline @Yolanda_Diaz_- & AGE & 24,74 \\
\hline @BeatrizSestayoD & PSdeG & 15,49 \\
\hline @ anaponton & BNG & 12,02 \\
\hline @jorqueracaselas & BNG & 11,81 \\
\hline @eva_solla & AGE & 10,78 \\
\hline @ carmeadan & BNG & 9,26 \\
\hline @DavidDeToen & AGE & 9,15 \\
\hline @JaviDorado & PP & 8,66 \\
\hline @xmBEIRAS & AGE & 4,52 \\
\hline @TereixaPaz & BNG & 3,71 \\
\hline @marisolsoneira & PSdeG & 3,16 \\
\hline @ AgustinCMATI & PP & 2,59 \\
\hline @ pachivazquez & PSdeG & 2,26 \\
\hline @ fran_caamano & PSdeG & 2,11 \\
\hline @ paulapra2 & PP & 1,6 \\
\hline @BalseiroPP & PP & 1,57 \\
\hline @ AntonSanchezG & AGE & 1,56 \\
\hline @jaimecastineira & PP & 1,4 \\
\hline @ CosmePombo & BNG & 1,24 \\
\hline @mblancoparadelo & PP & 1,23 \\
\hline @montseprado & BNG & 1,12 \\
\hline & &
\end{tabular}

Tabla 1. Diputados con mayor volumen de publicaciones diarias. Elaboración propia.

Yolanda Díaz (@Yolanda_Diaz_) se sitúa a la cabeza de los representantes autonómicos con mayor actividad en su perfil en Twitter. Con un promedio de 24,74 publicaciones día, la viceportavoz de AGE cuenta con un patrón de actividad superior a otros parlamentarios con 
mayor repercusión en la arena virtual como Xose Manuel Beiras (@xmBEIRAS, quien cuenta con un volumen de publicaciones de 8,66 tuits diarios), Javier Dorado (@JaviDorado, con una frecuencia de publicación de 12,03 tuits al día), Francisco Jorquera (@jorqueracaselas con una media de 11,81 tuits diarios), Ana Pontón (@anaponton, con 12,02 tuits/día). Por detrás de la portavoz suplende de AGE y líder de Izquierda Unida-Esquerda Unida, se sitúa la diputada gallega Beatriz Sestayo con un volumen de publicación de 15,49 tuits/día.

Las diferencias existentes entre el volumen de publicaciones día y la influencia de estos perfiles de políticos en Twitter (aspecto que será tratado en el siguiente apartado) subrayan que la frecuencia de actualización no conlleva -necesariamente- una mayor repercusión de la cuenta entre la ciudadanía digital si bien, prácticamente todos los perfiles de mayor repercusión (excepto @Feijoo2012) se encuentran entre los más actualizados.

En la lista de diputados con mayor volumen de publicaciones/día también resulta destacable el caso de @pachivazquez pese a la ausencia de actividad desde abril de 2013. Esta circunstancia remite a un elevado volumen de publicación durante la etapa pre y post electoral que todavía repercute en la media porcentual de tuits/día.

Atendiendo a los datos por grupos parlamentarios se vuelve a poner de manifiesto el mayor peso de representantes del BNG y de AGE entre los parlamentarios con mayor número de publicaciones/día.

Otra forma de cuantificar la actividad de un perfil de Twitter de especial interés desde la perspectiva de la participación de la ciudadanía es el porcentaje de menciones o respuestas. Se trata de citas a otros usuarios de Twitter de modo que su uso constituye un intento de implicación de éstos en la charla generada en torno a un determinado perfil.

Los perfiles de parlamentarios gallegos en Twitter efectúan un uso muy limitado de las menciones. Solamente Xosé Sánchez Bugallo (@X_S_Bugallo) presenta un promedio de menciones superior a 1. Esto es, que en cada una de sus publicaciones incorpora de media más de una mención a una cuenta-usuario.

\section{De 0,75 y más}

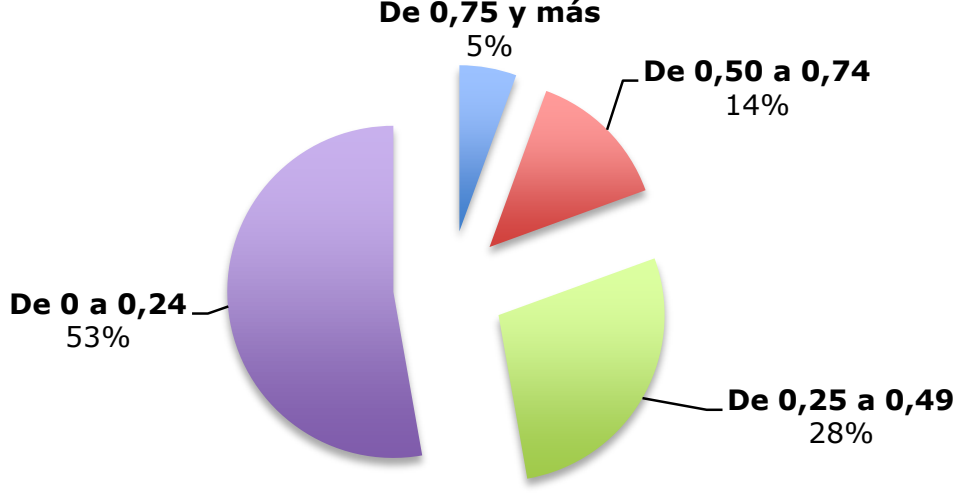

Gráfico 4. Perfiles de diputados en función de la media de menciones a otras cuentas-usuarios por publicación. Elaboración propia.

Como se puede observar en el gráfico 4, la mayoría de los perfiles apenas utilizan las menciones en sus publicaciones, tres de estas cuentas incluso no han utilizado nunca una mención, lo que implica una infrautilización de esta posibilidad de establecer interacción que ofrece la plataforma.

Si se atiende al porcentaje de contestaciones sobre el total de tuits (a mayor porcentaje mayor es la interacción con los usuarios de esta plataforma de microblogging), vuelve a destacar el mayor volumen de respuestas aportadas por el diputado Xosé Sánchez Bugallo. Con un $64 \%$ de contestaciones, este diputado del PSdeG-PSOE se sitúa a la cabeza de los parlamentarios que más feedback ofrece a los usuarios, seguido de Javier Dorado del PPdeG (@javidorado, con un 37\%) y al a Daniel Rodas del BNG (@DrodasRodas con un índice de respuesta de $35 \%$ ).

La diferencia de respuestas y menciones que presenta el perfil de @X_S_Bugallo, respecto a otros miembros de la Cámara gallega puede estar relacionado con su papel en la política local. En su presentación, el perfil de Xosé Sánchez Bugallo, hace referencia únicamente a su rol de portavoz del grupo socialista en el ámbito municipal de Santiago.

Asimismo, la comunidad de seguidores de dicho diputado es reducida, de manera que además de facilitar la interacción permite una mayor implicación de los usuarios con el político.

En lo que respecta a @JaviDorado (situado en el segundo y tercer puesto en volumen de contestaciones y menciones respectivamente), se trata de uno de los diputados que mejor se ha adaptado a esta plataforma de difusión. Una plataforma desde la que no sólo difunde sino que «escucha» a sus seguidores en Twitter, en su mayoría (al igual que el diputado) pertenecientes a las generaciones de nativos digitales. 


\begin{tabular}{|l|l|l|}
\hline Perfil & Partido & Contestación \\
\hline @X_S_Bugallo & PSdeG & $64 \%$ \\
\hline @JaviDorado & PP & $37 \%$ \\
\hline @DrodasRodas & BNG & $35 \%$ \\
\hline @ paulapra2 & PP & $26 \%$ \\
\hline @jaimecastineira & PP & $24 \%$ \\
\hline @ BeatrizSestayoD & PSdeG & $24 \%$ \\
\hline @ mhpm40 & PP & $20 \%$ \\
\hline @ VicenteDocasar & PSdeG & $17 \%$ \\
\hline @pachivazquez & PSdeG & $15 \%$ \\
\hline @fran_caamano & PSdeG & $15 \%$ \\
\hline
\end{tabular}

Tabla 2. Diputados con mayor volumen de respuesta a los usuarios. Elaboración propia.

Si se atiende a los restantes parlamentarios con un alto porcentaje de respuesta, se puede señalar que el feedback es mayor en el caso de aquellos perfiles de diputados de los partidos mayoritarios en la Cámara gallega.

\section{Influencia}

La presencia de los diputados en Twitter constituye una oportunidad para ampliar la proximidad de estos representantes a la ciudadanía gallega así como para estimular la participación. En este sentido, la repercusión o influencia de estos perfiles en la plataforma social pueden entenderse en términos de buen aprovechamiento de dicha potencialidad.

En una primera aproximación se analizó la repercusión de los diputados en lo que respecta a su número de seguidores. 


\begin{tabular}{|c|c|c|c|}
\hline NOMBRE & PART. & CUENTA & FOLLOWERS \\
\hline Beiras Torrado, Xosé Manuel & AGE & @xmBEIRAS & \begin{tabular}{|l|}
26.238 \\
\end{tabular} \\
\hline Núñez Feijóo, Alberto & PP & @Feijoo2012 & 6.058 \\
\hline Díaz Pérez, Yolanda & AGE & @YYolanda_Diaz_ & 5.661 \\
\hline Dorado Soto, Javier & PP & @ JaviDorado & 5.123 \\
\hline Jorquera Caselas, Francisco & BNG & @ jorqueracaselas & 4.757 \\
\hline Vázquez Fernández, Manuel & PSdeG & $@$ pachivazquez & 4.200 \\
\hline Pontón Mondelo, Ana Belén & BNG & @anaponton & 2.717 \\
\hline Sestayo Doce, Beatriz & PSdeG & $@$ BeatrizSestayoD & 1.682 \\
\hline Adán Villamarín, Carme & BNG & @ carmeadan & 1.314 \\
\hline Fernández Calviño, David & AGE & $@$ DavidDeToen & 1.284 \\
\hline Prado del Río, Paula & PP & $@$ paulapra2 & 1.059 \\
\hline Caamaño Domínguez, Francisco & PSdeG & $@$ fran_caamano & 1.042 \\
\hline Solla Fernández, Eva & AGE & $@$ eva_solla & 887 \\
\hline Fajardo Recouso, Juan Manuel & AGE & @ JMFajardoEU & 829 \\
\hline Sánchez García, Antón & AGE & @ AntonSanchezG & 809 \\
\hline Castiñeira Broz, Jaime & PP & @jaimecastineira & 790 \\
\hline $\begin{array}{l}\text { Hernández Fernández de Rojas, } \\
\text { Agustín }\end{array}$ & PP & @ AgustinCMATI & 777 \\
\hline Prado Cores, María Montse & BNG & @ montseprado & 719 \\
\hline Paz Franco, Tereixa & BNG & @TereixaPaz & 719 \\
\hline García García, Pablo & PSdeG & $@$ @ pablopsdeg & 713 \\
\hline Sánchez Bugallo, Xosé Antonio & PSdeG & @X_S_Bugallo & 654 \\
\hline Rueda Valenzuela, Alfonso & PP & @PP_AlfonsoRueda & 597 \\
\hline Soneira Tajes, María Soledad & PSdeG & @ marisolsoneira & 411 \\
\hline Martínez García, Consuelo & AGE & @Chelo_Martinez_ & 397 \\
\hline Balseiro Orol, José Manuel & PP & @BalseiroPP & 396 \\
\hline Blanco Paradelo, Moisés & PP & @ mblancoparadelo & 310 \\
\hline Vázquez Díaz, Ramón & AGE & @ ramonvazquezdia & 309 \\
\hline Docasar Docasar, Vicente & PSdeG & @ VicenteDocasar & 257 \\
\hline Pombo Rodríguez, Cosme & BNG & $@$ CosmePombo & 250 \\
\hline Méndez Romeu, José Luis & PSdeG & @mendezromeu & 231 \\
\hline Rodríguez González, Román & PP & $@$ RomanLalin & 162 \\
\hline Vilán Lorenzo, Patricia & PSdeG & $@$ patrivilan & 32 \\
\hline Rodas Chapela, Daniel & BNG & @DrodasRodas & 22 \\
\hline Pouso Maneiro, María Herminia & PP & $@$ mhpm40 & 21 \\
\hline Novoa López, Enrique & PP & @polynovoa & 2 \\
\hline
\end{tabular}

Tabla 3. Diputados en función del número de seguidores (followers). Elaboración propia. 
A partir del número de seguidores puede ratificarse el peso que -en el contexto de Twitter- adquieren los candidatos a la Xunta de los cuatro partidos que componen la Cámara gallega, todos ellos presentes entre los seis primeros perfiles con mayor número de seguidores.

En este sentido, resulta destacable la comunidad de seguidores del líder de AGE, Xosé Manuel Beiras, que dota a este perfil de mayor entidad en el ámbito de la Tuitesfera. Los 26.238 seguidores de @xmBEIRAS incluso superan la suma de las comunidades de los restantes líderes de partidos con presencia en la cámara (6.058 followers de @Feijoo2012; 5.661 de @Yolanda_Diaz_; 4.757 de @jorqueracaselas y 4.200 de @pachivazquez).

Entre los perfiles con mayor número de seguidores de la muestra también se encuentra Javier Dorado (PPdeG). Con sus 5.123 followers, el parlamentario más joven en la Cámara y más veterano en Twitter presenta la cuarta comunidad más extensa, cuestión que puede vincularse a su papel de Presidente de Novas Xeracións y Secretario General de Nuevas Generaciones (ámbito gallego y nacional respectivamente) dos organizaciones con gran peso en las redes sociales.

Además del tamaño de la comunidad se ha utilizado otro indicador para determinar la influencia de los perfiles en Twitter: el Klout. Tomando como referencia dicho índice, el diputado gallego con mayor influencia en el ámbito de la twitteresfera es Xosé Manuel Beiras (@xmBEIRAS). El portavoz de AGE en el Parlamento Gallego, con un índice Klout de 61 (a 5 de noviembre de 2013), ha logrado situarse por encima de diputados con mayor trayectoria en esta red social como @javidorado (en twitter desde 2009) 0 @jorqueracaselas (con perfil activo desde 2011).

\begin{tabular}{|l|l|l|}
\hline CUENTA & PART. & KLOUT \\
\hline @xmBEIRAS & AGE & 61 \\
\hline @JaviDorado & PP & 59 \\
\hline @Yolanda_Diaz_ & AGE & 56 \\
\hline @anaponton & BNG & 54 \\
\hline @jorqueracaselas & BNG & 54 \\
\hline @ carmeadan & BNG & 51 \\
\hline @paulapra2 & PP & 50 \\
\hline @eva_solla & AGE & 50 \\
\hline @Feijoo2012 & PP & 49 \\
\hline @AntonSanchezG & AGE & 49 \\
\hline
\end{tabular}

Tabla 4. Diputados más influyentes por grupo parlamentario. Elaboración propia.

En este sentido podría considerarse que su perfil de político histórico, su carisma o sus confrontaciones con el Presidente del Ejecutivo gallego en la Cámara, han logrado traspasar su influencia de la arena política real a la virtual, como ya se podía observar en su comunidad de 26.238 seguidores. No obstante, limitarse a esta consideración ofrecería una visión reducida de este fenómeno.

Entre los ocho perfiles de diputados que presentan un índice Klout igual o superior a 50, se puede destacar la presencia de dos representantes de AGE en el Parlamento de Galicia. Esto es, el 25\% de los diputados de esta coalición de izquierdas con presencia en Twitter.

Si a dichos diputados se les unen los tres perfiles del BNG con mayor influencia en Twitter (que suponen el $43 \%$ de los diputados de esta fuerza política en el Parlamento de Galicia), la hipótesis de que son los partidos minoritarios los que han logrado una apropiación más efectiva de esta plataforma de microblogging se refuerza.

Si bien la mayor influencia de los diputados de estos grupos parlamentarios podría estar determinada por la existencia de una polarización hacia la izquierda de la ciudadanía digital en España, señalada por Robles y Marco (2011), esta particularidad tendría que reflejarse también en la repercusión de los perfiles de diputados del PSdeG-PSOE como fuerza mayoritaria de izquierdas. No obstante, entre los perfiles de diputados gallegos con mayor índice Klout no se encuentra ningún representante de este grupo parlamentario.

En términos de influencia, el primer perfil de una diputada socialista (@BeatrizSestayoD) se encuentra en el úndecimo lugar, se impone al candidato de su partido para la Xunta de Galicia, @pachivazquez que cuenta con una comunidad de seguidores más amplia.

Ni siquiera el efecto candidato ha logrado situar a Pachi Vázquez (@pachivazquez) entre los diputados con mayor repercusión en el ámbito digital; un efecto que favorece ostensiblemente al perfil de Alberto Núñez Feijoo. La cuenta @feijo2012, de carácter electoral, todavía se sitúa entre los diez primeros perfiles en cuanto a su influencia aún sin registrar actividad desde octubre del pasado año (Klout se actualiza diariamente).

Además del Klout, otras variables que puede aportar información relativa a la influencia de un determinado perfil son los retuits y favoritos; dos fórmulas que expanden las publicaciones más allá de las fronteras del perfil con lo que amplían su repercusión. 


\begin{tabular}{|l|l|l|}
\hline Perfil & Partido & $\begin{array}{l}\text { \% de Tweets } \\
\text { Retwitteados }\end{array}$ \\
\hline @PP_AlfonsoRueda & PP & $100 \%$ \\
\hline @fran_caamano & PSdeG & $66,80 \%$ \\
\hline @ pachivazquez & PSdeG & $60,46 \%$ \\
\hline @Feijoo2012 & PP & $52,86 \%$ \\
\hline @BalseiroPP & PP & $50,99 \%$ \\
\hline @pablopsdeg & PSdeG & $49,48 \%$ \\
\hline @ramonvazquezdia & AGE & $48,15 \%$ \\
\hline @ AgustinCMATI & PP & $44,86 \%$ \\
\hline @montseprado & BNG & $42,52 \%$ \\
\hline @RomanLalin & PP & $42 \%$ \\
\hline
\end{tabular}

Tabla 5. Diputados con mayor volumen de tuits-retuiteados. Elaboración propia.

El mayor porcentaje de tuits retuiteados por otros lo detenta Alfonso Rueda, Secretario General del PPdeG, cuyo perfil @PP_AlfonsoRueda (con un $100 \%$ de tuits retuiteados) solamente cuenta con tres publicaciones.

El segundo perfil con mayor número de retuits es el de Francisco Camaño con $66,80 \%$ de publicaciones que han sido retuiteadas por otros. En el caso de estos usuarios, al igual que los siguientes (Pachi Vázquez y Alberto Núñez Feijoo), el elevado porcentaje de retuits se pude vincular a su carácter de líderes de partido, y por tanto se presume la existencia de unos usuarios implicados en difundir lo que publican para incrementar su alcance.

En este sentido resulta destacable la no presencia los líderes de AGE en esta lista de usuarios con mayor número de retuits. Si se parte del hecho de que tanto @xmBeiras como@Yolanda_Diaz_se encuentran entre los diputados con mayor repercusión, se puede considerar que en el ámbito de la tuitesfera los diputados gallegos con mayor número retuits no son los que generan mayor engagement en la Red.

\begin{tabular}{|l|l|l|}
\hline Perfil & Partido & $\begin{array}{l}\text { \% de Tuits } \\
\text { Favoritos }\end{array}$ \\
\hline @Feijoo2012 & PP & $35,71 \%$ \\
\hline @xmBEIRAS & AGE & $34,96 \%$ \\
\hline @PP_AlfonsoRueda & PP & $33,33 \%$ \\
\hline @fran_caamano & PSdeG & $28,00 \%$ \\
\hline @ AgustinCMATI & PP & $22,67 \%$ \\
\hline @VicenteDocasar & PSdeG & $21 \%$ \\
\hline @DrodasRodas & BNG & $20 \%$ \\
\hline @JaviDorado & PP & $18,40 \%$ \\
\hline @JMFajardoEU & AGE & $17,87 \%$ \\
\hline @ramonvazquezdia & AGE & $15,34 \%$ \\
\hline
\end{tabular}

Tabla 6. Diputados con mayor volumen de tuits marcados como favoritos. Elaboración propia.

En el caso del volumen de favoritos, pese a que el liderazgo del perfil @Feijo02012 o de @PP_AlfonsoRueda parecen reiterar los resultados de la tabla 5, resulta preciso destacar la mayor presencia de perfiles de grupos parlamentarios minoritarios.

En cualquier caso, retuits y favoritos constituyen dos indicadores que suelen tener mayor incidencia en aquellos contenidos de comunicación electoral o propaganda política (de gran relevancia para los afiliados quiénes colaboran en la difusión) que la información de interés de la Cámara o las llamadas a la participación de la ciudadanía gallega.

\section{A modo de conclusión}

El estudio llevado a cabo ha permitido determinar que un año después de la constitución de la actual Cámara gallega no parece existir una estrategia definida de diputados y grupos parlamentarios gallegos para integrar la participación de la ciudadanía digital a su actividad. Una 
situación que resulta especialmente patente en los grupos parlamentarios con mayor peso en la Cámara gallega cuya representación en la plataforma social es limitada tanto por el número de perfiles como por su repercusión.

De hecho, se han podido destacar cuatro características comunes a la actividad de los diputados del Grupo Popular y Socialista en el ámbito de esta red social: 1) menor porcentaje de parlamentarios con perfil en Twitter, 2) algunos de ellos creados ex profeso para la campaña electoral de otoño de 2012 (que solamente han tenido actividad durante la misma), 3) mayor volumen de cuentas inactivas y 4) la prolongada ausencia de los portavoces parlamentarios de esta plataforma social (que recientemente ha subsanado el grupo socialista), lo que implica la ausencia de unas fuentes valiosas de información para la tuitesfera gallega.

La limitada presencia de perfiles de los diputados gallegos en Twitter se sitúa en la línea de estudios anteriores como el de Túñez y Sixto (2011) que destacaba la escasa implantación de las redes sociales entre los miembros del Congresos de los Diputados, dada la falta de confianza de los representantes estatales en las potencialidades de estas plataformas.

En el caso de los diputados gallegos resulta complejo determinar si se trata de desconfianza hacia las redes sociales o si, por el contrario, refleja una falta de percepción de su necesidad en el contexto actual para facilitar la interacción con la ciudadanía digital. El análisis de retuits y favoritos, por ejemplo, no sólo ha puesto de relevancia el escaso interés de los asuntos tratados en las publicaciones de los diputados gallegos en la tuitesfera, sino incluso la creación de comunidades «no-espontáneas» en torno a algunos líderes políticos. El éxito que tienen las publicaciones de perfiles como el de @PP_AlfonsoRueda o @fran_caamano remite a una comunidad de internautas implicados en la difusión de sus contenidos más que en la participación e interacción con dichas cuentas, lo que parece obedecer a una difusión orquestada.

Más allá de los perfiles de diputados del Parlamento de Galicia gestionados de forma muy básica, el estudio desarrollado ha destacado la existencia de otras cuentas que tienen una actividad destacable que se enriquece con las aportaciones de los usuarios.

Frente a la ausencia de una estrategia clara de los parlamentarios autonómicos populares y socialistas en Twitter, se puede hacer referencia a una interesante apropiación de esta plataforma 2.0 por parte de los diputados de AGE y BNG. Prácticamente todos los representantes de estos dos grupos cuentan con un perfil activo en la plataforma de microblogging, la mayoría con gran influencia en la tuitesfera gallega.

La diferente utilización de esta red social por parte de los grupos con mayor y menor representación en la Cámara refuta la aseveración de Padró-Solanet y Carenal (2008). En este caso, los partidos grandes, establecidos y con más recursos son los que sacan un menor provecho de Twitter.

Quizás durante la campaña electoral hayan podido destinar más recursos a la gestión de las redes sociales (que explica la influencia del @Feijo02012, tras un año de inactividad) pero durante el día a día parlamentario la disciplina de partido y la excesiva jerarquización -unidas a la ausencia de un portavoz legitimado en el ámbito 2.0- suponen un freno a la actividad de los diputados en sus perfiles en esta plataforma social.

En este caso, frente a la hipótesis de la igualación (que se basa en la posibilidad de un igual acceso a las TICs y a las plataformas 2.0 de partidos clásicos, mayoritarios, establecidos y de aquellos minoritarios, recién creados), se puede decir que Twitter ha permitido una mayor influencia de las fuerzas políticas minoritarias, al contar con una mayor implicación de sus miembros a la hora de participar y fomentar la participación en la redes sociales.

La dupla tendencia señalada en la adopción de Twitter por los grupos parlamentarios gallegos puede explicarse a través de los dos modelos de apropiación de las nuevas tecnologías de Friseen (2003, citado en Mari Saez 2010). Si bien el uso que los miembros del PPdeG y el PSdeG-PSOE en la Cámara gallega dan a Twitter responde a un enfoque instrumental -como herramienta- la incorporación de esta plataforma de microblogging a la actividad parlamentaria y mediadora de AGE y BNG se aproximaría más a un enfoque orgánico, como una nueva arena de expansión en la que iniciar nuevas actividades y relaciones con la ciudadanía.

Otra cuestión reseñable es la repercusión alcanzada por el perfil de : @xmBeiras. Frente a la escasa adaptación e influencia de otros líderes políticos de grupos parlamentarios gallegos, Xosé Manuel Beiras ha logrado traspasar su liderazgo carismático a la Red, como se refleja en Twitter.

A pesar de lo reciente de su perfil (creado para la cita electoral), el perfil de Xosé Mauel Beiras en Twitter ha logrado aglutinar a una amplia comunidad de seguidores, y detenta un liderazgo indiscutible en lo que se refiere a la influencia en dicha red social.

Este liderazgo puede relacionarse con la presidencialización de las instituciones políticas y sus efectos en el refuerzo de la autonomía política del líder de la formación partidista y en la notoriedad que adquiere en el contexto de la ciudadanía (Criado y Martínez Fuentes, 2010). Una notoriedad que @xmBeiras ha sabido canalizar también en la arena virtual.

\section{Bibliografía}

Aggio, C. (2010). Campanhas online: O percurso de formação das questões, problemas e configurações a partir da literatura produzida entre 1992 e 2009. Opiniao Publica, 16(2), 426-445.

Ampofo, L., Anstead, N., y O'Loughlin, B. (2011). Trust, confidence, and credibility: Citizen responses on twitter to opinion polls during the 2010 UK general election. Information Communication and Society, 14 (6), 850-871.

Andrew, A. M. (2009). Internet commentary: Cybernetics and systems on the web: Cybernetics and the white house. Kybernetes, 38(9), $1433-1435$ 
Anduiza, E.; Cantijoch, M.; Colombo, C.; Gallego, A.y Salcedo, J. (2010). Los usos políticos de Internet en España. Reis, Revista Española de Investigaciones Sociológicas, 129, 133-146.

Anduiza, E.; Cantijoch, M.; Colombo, C.; Gallego, A.y Salcedo, J. (2010). Internet y participación política en España. Opiniones y actitudes, $n^{0}$ 63. Madrid: Centro de investigaciones sociológicas.

Araya Dujisin, R.(2005). Internet, política y ciudadanía. Nueva Sociedad, 195, 56-71.

Arnstein, S. R (1969). A Ladder of Citizen Participation, JAIP, 35 (4), 216-224. En http://lithgow-schmidt.dk/sherry-arnstein/ladder-of-citizenparticipation.html (12/06/2013).

Baumgartner, J. C. y Morris, J. S. (2010) MyFaceTube Politics SocialNetworking Web Sites and Political Engagement of Young Adults. Social Science Computer Review, 28 (1), 24-44. ftp://24.166.108.44/TAU/Campaigns\%20and\%20Elections/Baumgarten.pdf

Baxter, G., Marcella, R., y Varfis, E. (2011). The use of the internet by political parties and candidates in Scotland during the 2010 UK general election campaign. Aslib Proceedings: New Information Perspectives, 63(5), 464-483.

Bermejo Acosta, F.(2003). Democracia electrónica, participación ciudadana y juventud. Estudios de Juventud, 61 (3), 51-57. En http://www.injuve.es/sites/default/files/NUM61_4art.pdf (12/06/2013).

Bimber, B. (1998). The Internet and political transformation: populism, community and pluralism. Polity, 31 (1), 133-160. En http://www.calstatela.edu/faculty/blawson/bimber\%201998.pdf (12/06/2013).

Bimber, B.; Flanagin, A. J.; Stohl, C, (2005). Reconceptualizing collective action in the Contemporary Media Environment. Communication Theory, 4, 3645-388. En http://ucsbsocialcomputing.pbworks.com/f/bimber,\%2Bet\%2Bal.pdf (12/06/2013).

Bimber, B.(1999). The Internet and citizen communication with government: Does the medium matter? Political Communication, 16 (4): $409-$ 428.

Borge, Rosa (2005). La participación electrónica: estado de la cuestión y aproximación a su clasificación. IDP. Revista de Internet, Derecho y Política, 1. UOC. En http://www.uoc.edu/idp/1/dt/esp/borge.pdf (12/06/2013).

Borge, R.; Colombo, C. y Welp, Y.(2008). Análisis explicativo de la participación ciudadana electrónica y presencial en el ámbito municipal de Cataluña IDP. Revista de Internet, Derecho y Política. 6. UOC En http://www.uoc.edu/idp/6/dt/esp/borge_colombo_welp.pdf (12/06/2013). Borins, S. (2009). From online candidate to online president. International Journal of Public Administration, 32(9), 753-758.

Casacuberta, D. y Gutiérrez-Rubí, A. (2010). E-participación: de cómo las nuevas tencologías están transformando la participación ciudadana. Razón y palabra, ${ }^{\circ}$ 73, 9 págs. En http://razonypalabra.org.mx/N/N73/MonotematicoN73/12-M73Casacuberta-Gutierrez.pdf (12/06/2013).

Cervi, E. U., y Massuchin, M. G. (2012). Social networks in 2010 election campaign: Analysis of Parana governor candidates' twitter postings. [Redes sociais como ferramenta de campanha em disputas subnacionais: Análise do twitter nas eleições para o governo do paraná em 2010] Sociedade e Cultura, 15(1), 25-38.

Cogburn, D. L., y Espinoza-Vasquez, F. K. (2011). From networked nominee to networked nation: Examining the impact of web 2.0 and social media on political participation and civic engagement in the 2008 Obama campaign. Journal of Political Marketing, 10(1-2), 189-213. Coleman, S. y Gotze, J. (2001). Bowling together: Online public engagement in Policy Deliberation. Londres: Hansard Society. En http://catedras.fsoc.uba.ar/rusailh/Unidad\%207/Coleman\%20and\%20Gotze\%20Bowling\%20Together,\%20online\%20public\%20engagement \%20in\%20policy\%20deliberation.pdf (12/06/2013).

Congosto, M.L.; Fernández, M. y Moro Egido, E. (2011). Twitter y política: Información, opinión y ¿Predicción?. Cuadernos de Comunicación Evoca, 4, 11-15. http://markov.uc3m.es/ emoro/ps/evoca.pdf

Cremonese, D. (2012). On-line politics: The use of twitter as a tool of social capital in the 2010 presidential elections. [Politica on-line: A utilização do Twitter como ferramenta de capital social nas eleições presidenciais de 2010] Sociedade e Cultura, 15(1), 135-149.

Criado, J. I. y Martínez Fuentes, G. (2011). Blogging político y personalizaciónde la democracia local en Españay Portugal. Evidencias presentes y propuestas de futuro. Estudios de Progreso, 56. En http://www.falternativas.org/estudios-de-progreso/documentos/documentosde-trabajo/blogging-politico-y-personalizacion-de-la-democracia-local-en-espana-y-portugal-evidencias-presentes-y-propuestas-de-futuro (12/06/2013).

De Landtsheer, C., Krasnoboka, N. y Neuner, C. (2001). La facilidad de utilización de los web sites de partidos políticos. Estudio de algunos países de Europa del Este y Occidental. CIC. Cuadernos de Información, 6, 107-140.

Gibson, R. y Cantijoch, M. (2011). Conceptualizing and measuring participation in the age of the internet: Is online political engagement really different to offline? Paper prepared for presentation at the Colloquium «Methodological Challenges in Participation Research», Cordoba, España, $\quad 4-5 \quad$ Noviembre $\quad$ de $2011 . \quad$ En http://drupals.humanities.manchester.ac.uk/ipol/sites/default/files/newdocs/Cordoba_2011.pdf (12/06/2013).

Gibson, R. y McAllister, I. (2005). Does Cyber Campaigning Win Votes? Online Communication in the 2004 Australian Election. CSD Working Paper En http://escholarship.org/uc/item/6tn8c287 (12/06/2013).

Gibson, R. y Ward, S. (2002). Virtual Campaigning: Australian Parties and the Impact of the Internet. Australian Journal of Political Science, 37 (1), 99-130. En http://www.tandfonline.com/doi/abs/10.1080/13603100220119047?journalCode=cajp20\#.UbRj1uv9-Hk (12/06/2013).

Gibson, R., Ward, S. y Lusoli, W.(2002). The internet and political campaigning: the new medium comes of age? Representation, 39(3), 166180 En http://lusoli.info/papers/gibson\%20et\%20al\%202003\%20representation.pdf (12/06/2013).

Gibson, R. y Römmele, S- (2007). Political Communication. En Caramani, Daniele (ed): Comparative politics. Oxford: Oxford University Press. En http://www.politikkampagnen.de/politikkampagnen/documents/pdf/c19-gibsonandroemmele_rg_28_04_07.pdf (12/06/2013).

Gil de Zuniga, H., Veenstra, A., Vraga, E. y Shah, D. (2010). Digital Democracy: Reimagining Pathways to Political Participation. Journal of $\begin{array}{lllllll}\text { Information, } & \text { Technology } & \text { y } & \text { Politics } & 7 & \text { (1), 36-51. }\end{array}$ https://webspace.utexas.edu/hg3985/www/documents/Gil\%20de\%20Zuniga,\%20Veenstra,\%20Vraga\%20\&\%20Shah\%20\%282010\%29\%20 Journal\%20of\%20Information\%20Technology\%20\&\%20Politics\%207\%281\%29,\%2036-51.pdf (12/06/2013).

González Esteban, J. L. (2010). La base electoral de Obama, redes sociales virtuales y reales: Los casos de generation engage y moms for Obama. Revista Mediterránea De Comunicación, (1), 25-35.

González Esteban, J. L. (2010). La base electoral de Obama, redes sociales virtuales y reales: Los casos de generation engage y moms for Obama. Revista Mediterránea De Comunicación, (1), 25-35.

González-Bailón, S. (2009). Redes y mecanismos de interdependencia. Desarrollos teóricos más allá de los modelos de acción racional. Revista Internacional de Sociología (RIS), 67 (3), 537-558.

Goodman, J., Wennerstrom, A., y Springgate, B. F. (2011). Participatory and social media to engage youth: From the Obama campaign to public health practice. Ethnicity and Disease, 21(3 SUPPL. 1), S194-S199. 
Hackers, K. y Van Dijk, J. (2000). Digital Democracy. Issues of Theory and Practice. Londres: Sage.

Lévy, P. (2004). Inteligencia colectiva: Por una antropología del ciberespacio. Washington: OPS.

Lusoli, W. (2005). A second-order médium? The Internet as a source of electoral information in 25 European countries. Information Polity, 10, 247-265.

Margolis, Michael; Resnick, David y Tu, Ching-Chang (1997). Campaigning on the Internet Parties and Candidates on the World Wide Web in the 1996 Primary Season. The International Journal of Press/Politics January, 2 (1), $59-78$

Margolis, Michael; Resnick, David y Wolfe, Joel D. (1999). Party Competition on the Internet in the United States and Britain. The International Journal of Press/Politics 4 (4), 24-47.

Mari Sáez, V. M. (2010). Crítica del concepto de capital social (Putnam) y propuesta del enfoque de capital informacional (Hamelink) para el análisis y el diseño de estrategias de apropiación social de las TIC por parte de los movimientos sociales. Revista de Economía Política de las Tecnologías de la Información y Comunicación, 10 (1), 16 págs. En http://www.seer.ufs.br/index.php/eptic/article/view/79/52 (12/06/2013).

Matud Juristo, Á. (2009). Política 2.0. algo más que internet. Nueva Revista De Política, Cultura y Arte, (121), 46-49.

Menéndez, M. A. (2011). Las redes sociales y su efecto político. ¿Nuevas Fuenteovejunas digitales? Telos, 89. En http://sociedadinformacion.fundacion.telefonica.com/DYC/TELOS/REVISTA/Dossier/DetalleArtculoTELOS_89TELOS_DOSSIER3/seccion=1 266\&idioma=es_ES\&id=2011102411390001\&activo=6.do (12/06/2013).

Padró-Solanet, A. y Cardenal, A. S. (2008). Partidos y política en Internet: Un análisis de los websites de los partidos catalanes. Revista de Internet, Derecho y Política, 6 , 46-64.

Reig, D. (2012). Disonancia cognitiva y apropiación de las TIC. Buscando los efectos de la participación. Telos, 90. En $\mathrm{http}: / /$ sociedadinformacion.fundacion.telefonica.com/DYC/TELOS/REVISTA/TribunasdelaComunicacin_90TELOS_TRIBUNA2/seccion=1213 \&idioma=es_ES\&id=2012020215200001\&activo=7.do (12/06/2013).

Resina de la Fuente, J. (2010). Ciberpolítica, , redes sociales y nuevas movilizaciones en España: el impacto digital en los procesos de deliberación y participación ciudadana. Mediaciones Sociales, 7, 143-164.

Robertson, S. P., Vatrapu, R. K. y Medina, R. (2010). Off the wall political discourse. Facebook use in the 2008 US presidential election. Information Polity, 15 (1-2), 11-31. En http://www.itu.dk/people/rkva/2011-Fall-T17/readings/JournalArticle-OffTheWallPoliticalDiscourse.pdf (12/06/2013).

Robles, J.M. y de Marco, S. (2011). La participación digital y el comportamiento político de los usuarios de internet. Un análisis descriptivo de la ideología de los internautas. Papeles del CEIC, 65. En: http://www.identidadcolectiva.es/pdf/65.pdf (12/06/2013).

Rueda Ortiz, Rocío (2009). Convergencia tecnológica: síntesis o multiplicidad política y cultural. Signo y pensamiento, 25 (54), 114-130.

Túñez, Miguel y Sixto, José (2011). Redes sociales, política y Compromiso 2.0: La comunicación de los diputados españoles en Facebook. eRevista Latina de Comunicación Social, 66, 210-246. En

http://www.revistalatinacs.org/11/art/930_Santiago/09_Tunez.html (12/06/2013).

Ward, S.; Lugosi, W. y Gibson, R. (2002). Virtually Participating: A Survey of Online Party Members. Information Polity, 7(4), 9-108. En http://www.lusoli.info/papers/ward_et_al_2002.pdf (12/06/2013).

Westling, M. (2007). Expanding the Public Sphere: The impact of Facebook on Political Communication. En http://www.thenewvernacular.com/projects/facebook_and_political_communication.pdf (12/06/2013). 